\title{
LINC0638 IncRNA is involved in the local recurrence of melanoma following surgical resection
}

\author{
WEIRONG XIAO ${ }^{1}$ and AIWAN YIN ${ }^{2}$ \\ Departments of ${ }^{1}$ Dermatology and ${ }^{2}$ Traditional Chinese Medicine, Hunan Provincial People's Hospital, \\ The First Affiliated Hospital of Hunan Normal University, Changsha, Hunan 410000, P.R. China
}

Received August 1, 2018; Accepted February 8, 2019

DOI: $10.3892 /$ ol.2019.10322

\begin{abstract}
Melanoma is a rare malignancy in China and the treatment outcomes are generally satisfactory. However, postoperative recurrence can be life-threatening. The current study aimed to investigate the involvement of long intergenic non-protein coding RNA 1638 (LINC01638) long non-coding RNA (lncRNA) in the recurrence of melanoma. Expression of LINC01638 lncRNA in skin biopsies and plasma of patients with melanoma, patients with benign skin lesions and healthy controls was detected by reverse transcription-quantitative polymerase chain reaction. Diagnostic values of LINC01638 lncRNA for melanoma were analyzed by receiver operating characteristic curve analysis. The association between LINC01638 lncRNA and clinicopathological data of patients with melanoma was analyzed by $\chi^{2}$ test. All patients were followed up for five years to record recurrence. LINC01638 lncRNA expression vectors and shRNAs were transfected into human melanoma cell lines and the effects of LINC01638 lncRNA overexpression and knockdown on cell proliferation were analyzed by cell counting kit- 8 assay. LINC01638 IncRNA was significantly upregulated in patients with melanoma compared with the other two groups of patients, and upregulation of LINC01638 lncRNA distinguished patients with melanoma from patients with benign skin lesions and healthy controls. LINC01638 lncRNA expression was significantly associated with tumor size but not with other patient clinical data. Plasma levels of LINC01638 IncRNA were further increased during follow-up in patients with local recurrence but not in patients without recurrence. LINC01638 lncRNA overexpression promoted, while knockdown inhibited proliferation of cells of melanoma cell lines, C32 and SK-MEL-28, in vitro. The upregulation of LINC01638 lncRNA was likely
\end{abstract}

Correspondence to: Dr Aiwan Yin, Department of Traditional Chinese Medicine, Hunan Provincial People's Hospital, The First Affiliated Hospital of Hunan Normal University, 61 Jiefang West Road, Changsha, Hunan 410000, P.R. China

E-mail: bonfo73@163.com

Key words: melanoma, long intergenic non-protein coding RNA 1638 long non-coding RNA, local recurrence associated with the local recurrence of melanoma following surgical resection.

\section{Introduction}

Melanoma is a rare type of malignant tumor in China; however, this disease still affects a considerable number of patients every year (1). Melanoma is the most aggressive form of skin cancer and it has an increasing incidence across the world (2). At present, there are no effective treatment strategies for melanoma in the advanced stages (3). Although surgical resection is considered as a radical treatment for melanoma in the early stages, survival of patients following surgery is still poor due to high recurrence rate (4). Previous studies have demonstrated that recurrent melanoma is closely associated with exposure to UV rays, smoking and lack of exercise $(5,6)$. However, the molecular mechanisms underlying recurrent melanoma remain to be elucidated $(5,6)$.

In addition to messenger RNAs encoding proteins, the human genome also transcribes a large set of non-coding RNAs, which serve roles in physiological processes and the development of human diseases (7). Long non-coding RNAs (lncRNAs) are a subgroup of non-coding RNAs composed of $>200$ nucleotides (8). IncRNAs are central regulators in cancer biology (9). Long intergenic non-protein coding RNA 1638 (LINC01638) is a recently identified lncRNA which serves an oncogenic role in triple-negative breast cancer (10). Preliminary microarray data revealed in the present study indicated that LINC01638 IncRNA is upregulated in melanoma tissues compared with healthy tissues (data not shown). The present study demonstrated the upregulation of LINC01638 lncRNA in melanoma and suggested that LINC01638 lncRNA may participate in the growth of melanoma and its local recurrence following surgical resection.

\section{Materials and methods}

Patients and specimens. The current study included 40 patients with melanoma and 23 patients with benign skin lesions diagnosed in Hunan People's Hospital (Changsa, China) between March 2011 and March 2013. The inclusion criteria were as follows: i) Patients were diagnosed with melanoma or benign skin lesions through pathological examinations; ii) patients were diagnosed for the first time and no treatment was received 
during the three months before the study; iii) patients were diagnosed with American Joint Committee on Cancer (AJCC) stage I-IIIA melanoma and did not have tumor metastasis; iv) patients understood the experimental protocol and were willing to participate; and v) patients complicated the whole procedure and five-year follow-up. The exclusion criteria were as follows: i) Patients with co-morbidities, including chronic inflammation; ii) patients transferred to other hospitals; and iii) patients who could not attend the follow-up or who succumbed prior to the confirmation of recurrence. There were 10 cases of stage I, 21 cases of stage II and 9 cases of stage IIIA melanoma. A total 16 healthy patients who were willing to donate skin biopsies and blood were included to serve as the control group. No significant differences in age and sex were identified between the three patient groups. Patient basic information is presented in Table I. Plasma and skin biopsy tissues were stored in liquid nitrogen prior to use. The present study was approved by the Ethics Committee of Hunan People's Hospital. Written informed consent was obtained from all patients prior to enrolment.

Treatment, blood extraction and follow-up. Blood $(5 \mathrm{ml})$ was collected on the day of admission. All patients with melanoma were treated with surgical resection, followed by chemotherapy. All patients were followed up for 5 years following discharge to record recurrence. Blood was collected again in cases of recurrence and at the end of follow-up period in cases of no recurrence. Plasma was prepared by centrifuging blood at $1,250 \mathrm{x} \mathrm{g}$ for $15 \mathrm{~min}$ in EDTA tubes at room temperature.

Reverse transcription-quantitative polymerase chain reaction ( $R T-q P C R)$. Following RNA extraction from plasma, biopsies and in vitro cultivated cells using TRIzol ${ }^{\circledR}$ reagent (Invitrogen; Thermo Fisher Scienfitic, Inc., Waltham, MA, USA), a NanoDrop ${ }^{\mathrm{TM}} 2000$ Spectrophotometer (Thermo Fisher Scientific, Inc., Pittsburgh, PA, USA) was used to measure RNA concentration. To enrich RNA concentration, RNA samples were mixed with alcohol and glycogen (1:1,000; Sigma-Aldrich; Merck KGaA, Darmstadt, Germany). Following incubation at $-80^{\circ} \mathrm{C}$ for $30 \mathrm{~min}$, the mixture was centrifuged at $4^{\circ} \mathrm{C}$ for $30 \mathrm{~min}$ at $12,000 \mathrm{x}$ g. RNA samples with an A260/A280 ratio of 1.8-2.0 were subjected to reverse transcription using SuperScript IV Reverse Transcriptase (Thermo Fisher Scientific, Inc.) to synthesize cDNA at $55^{\circ} \mathrm{C}$ for $30 \mathrm{~min}$ and $75^{\circ} \mathrm{C}$ for $10 \mathrm{~min}$. All PCR reactions were prepared using SYBR ${ }^{\circledR}$ Green Real-Time PCR Master Mixes (Thermo Fisher Scientific, Inc.) and the following primers: 5'-AATACATCA GCACTGTTGCCTTT-3' (forward) 5'-CTCCATACATAC ATCTCCAAAAAGT-3' (reverse) for LINC01638 IncRNA; 5'-GACCTCTATGCCAACACAGT-3' (forward) and 5'-AGT ACTTGCGCTCAGGAGGA-3' (reverse) for $\beta$-actin. The following thermocycling conditions were used for the PCR: $95^{\circ} \mathrm{C}$ for $1 \mathrm{~min} ; 40$ cycles of $95^{\circ} \mathrm{C}$ for $12 \mathrm{sec}$ and $58.5^{\circ} \mathrm{C}$ for $40 \mathrm{sec}$. Data normalization was performed using the $2^{-\Delta \Delta \mathrm{Cq}}$ method (11) with $\beta$-actin as the internal reference gene. PCR products were sequenced to ensure the correct products were obtained.

Cell lines and cell culture. A normal human skin cell line CCD-1059Sk and two human melanoma cell lines C32 and
SK-MEL-28 were obtained from the American Type Culture Collection (ATCC; Manassas, VA, USA). The cells were cultured with Eagle's Minimum Essential Medium with $10 \%$ fetal bovine serum (Sangon Biotech Co., Ltd., Shanghai, China) at $37^{\circ} \mathrm{C}$ with $5 \% \mathrm{CO}_{2}$. Vectors containing full length LINC01638 lncRNA, short hairpin RNA (shRNA) (5'-GGC CCTCCTGCTGATGAGAGAC-3') and negative control shRNA (5'UUCUCCGAACGUGUCACGUGGC-3) were purchased from GeneCopoeia Inc. (Rockville, MD, USA). LINC01638 expression vector (15 nM) and shRNAs (20 nM) were transfected into cells using Lipofectamine ${ }^{\circledR} 3000$ according to the manufactuer's protocol (Thermo Fisher Scientific, Inc.). Transfection with empty vectors or negative control shRNA was used as negative control. Untransfected cells were used as control cells. LINC01638 lncRNA expression was detected by RT-qPCR $12 \mathrm{~h}$ following transfection.

Cell proliferation assay. Following transfection (24 h later), a cell proliferation assay was performed to determine the proliferation in cells with an LINC01638 overexpression rate $>200 \%$ or knockdown rate $<50 \%$. The cell counting kit- 8 (CCK-8; Sigma-Aldrich; Merck KGaA) was used to detect cell proliferation. Briefly, cells were harvested, and cell suspensions were prepared with a density of $4 \times 10^{4}$ cells $/ \mathrm{ml}$. Each well of a 96-well plate was filled with $0.1 \mathrm{ml}$ cell suspension. Cells were cultured at $37^{\circ} \mathrm{C}$ in a $5 \% \mathrm{CO}_{2}$ incubator, followed by addition of $10 \mu \mathrm{l} \mathrm{CCK}-8$ solution after 24, 48, 72 and $96 \mathrm{~h}$. Cells were cultured for additional $4 \mathrm{~h}$ and optical density values were measured using a microplate reader (Bio-Rad Laboratories, Inc., Hercules, CA, USA) at a wavelength of $450 \mathrm{~nm}$.

Statistical analysis. GraphPad Prism software (version 6; GraphPad Software Inc., La Jolla, CA, USA) was used for all statistical analyses. Data are presented as the mean \pm standard deviation. Comparisons between two groups were performed by unpaired t- test. Comparisons between two time points within the same group were performed by paired t-test. Comparisons among multiple groups were performed by one way analysis of variance followed by a Tukey test. Associations between LINC01638 lncRNA and clinicopathological data of patients with melanoma were analyzed by the $\chi^{2}$ test. Receiver operating characteristic (ROC) curve analysis was performed to evaluate the diagnostic value of LINC01638 for melanoma. $\mathrm{P}<0.05$ was considered to indicate a statistically significant difference.

\section{Results}

LINC01638 IncRNA is significantly upregulated in patients with melanoma. Expression of LINC01638 lncRNA in skin biopsies and plasma samples of patients with melanoma, patients with benign skin lesions and healthy controls was quantified using RT-qPCR. Expression of LINC01638 lncRNA in skin biopsies was significantly upregulated in patients with melanoma compared with the other two patient groups ( $\mathrm{P}<0.05$; Fig. 1A). In addition, plasma levels of LINC01638 lncRNA were significantly increased in patients with melanoma compared with the other two patient groups $(\mathrm{P}<0.05$; Fig. 1B). By contrast, there were no significant differences in expression of LINC01638 lncRNA in skin biopsies (Fig. 1A) 
Table I. Basic information of the three groups of patients.

\begin{tabular}{lcccc}
\hline & \multicolumn{2}{c}{ Sex } & & \\
\cline { 2 - 3 } Group & Male & Female & Age range (years) & Average age (years) \\
\hline Melanoma & 22 & 18 & $27-66$ & $46.1 \pm 5.3$ \\
Benign skin lesion & 12 & 11 & $24-67$ & $45.9 \pm 6.0$ \\
Control & 7 & 9 & $22-68$ & $46.2 \pm 5.2$ \\
\hline
\end{tabular}
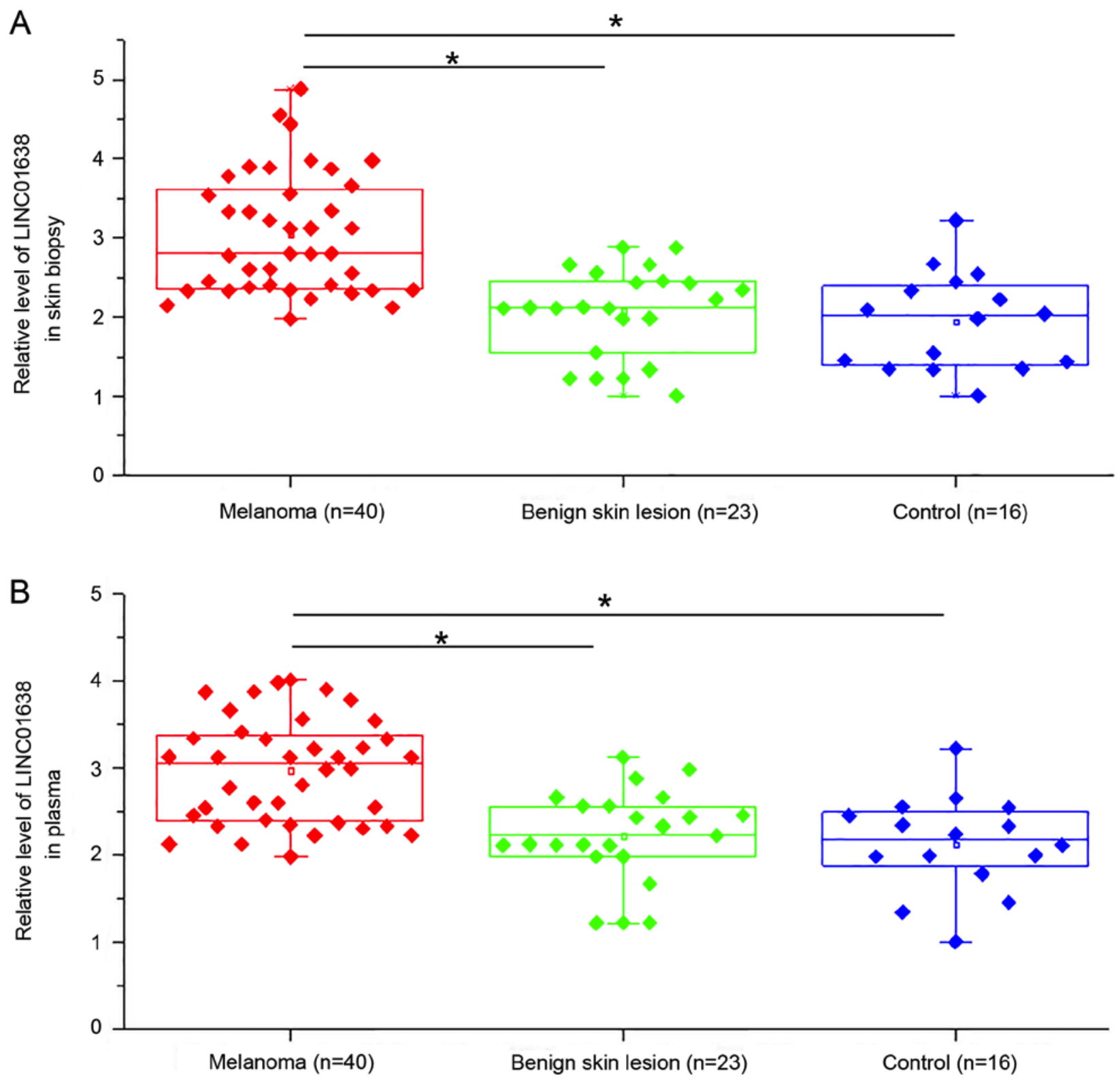

Figure 1. LINC01638 lncRNA is significantly upregulated in patients with melanoma. LINC01638 lncRNA expression was significantly upregulated in (A) skin biopsies and (B) plasma of patients with melanoma compared with patients with benign skin lesions and healthy controls. * $\mathrm{P}<0.05$. LINC01638, long intergenic non-protein coding RNA 1638; lncRNA, long non-coding RNA.

and plasma (Fig. 1B) between patients with benign skin lesions and healthy controls. Slightly increased LINC01638 lncRNA expression in both biopsies and plasma was observed with increasing clinical and tumor stages (no statistical significance; data not shown).
Upregulation of LINC01638 lncRNA distinguishes patients with melanoma from the other patient groups. Diagnostic values of LINC01638 lncRNA for melanoma were analyzed by receiver operating characteristic curve analysis. For LINC01638 lncRNA expression in skin biopsies compared 

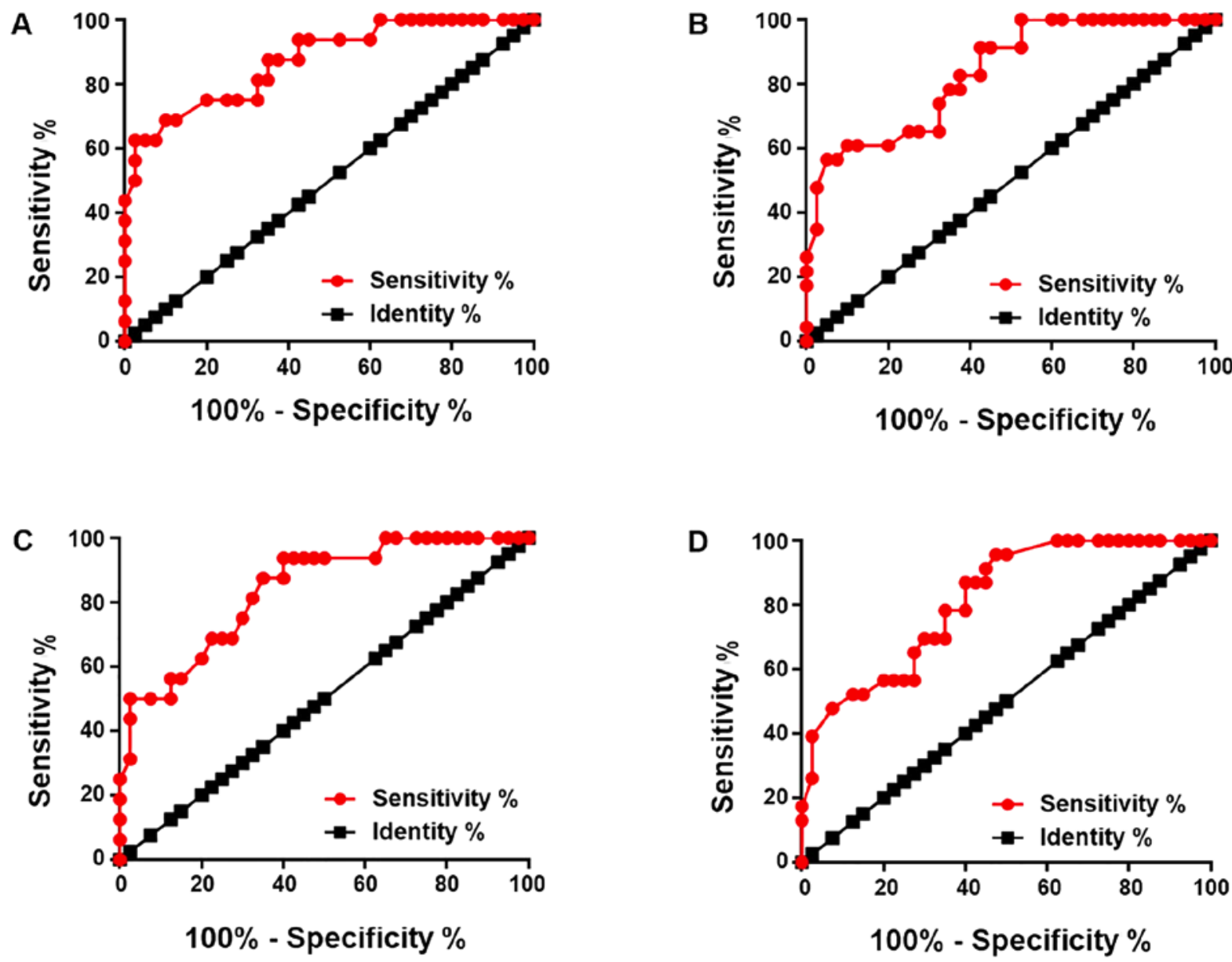

Figure 2. Upregulation of LINC01638 lncRNA distinguishes patients with melanoma from other the other two patient groups. Receiver operating characteristic curve analysis of the diagnostic value of LINC01638 lncRNA expression in skin biopsies for patients with melanoma compared with (A) healthy controls and (B) patients with benign skin lesions as references, and the diagnostic value of LINC01638 lncRNA expression in plasma for patients with melanoma compared with (C) healthy controls and (D) patients with benign skin lesions as references. LINC01638, long intergenic non-protein coding RNA 1638; lncRNA, long non-coding RNA.

with healthy controls as references, the area under the curve (AUC) was 0.8734 , with a $95 \%$ confidence interval (CI) of $0.7699-0.9700$ and standard error of $0.05281(\mathrm{P}<0.0001$; Fig. 2A); with patients with benign skin lesions as references, the AUC was 0.8370 , with a $95 \%$ CI of $0.7380-0.9359$ and standard error of 0.05049 ( $\mathrm{P}<0.0001$; Fig. 2B). For LINC01638 lncRNA expression in plasma, with healthy controls as references, the AUC was 0.8391, with a 95\% CI of 0.7287-0.9494 and standard error of 0.05269 ( $\mathrm{P}<0.0001$; Fig. $2 \mathrm{C})$; with patients with benign skin lesions as references, the AUC was 0.8136, with a 95\% CI of 0.7097-0.9174 and standard error of 0.05298 (P<0.0001; Fig. 2D).

LINC01638 lncRNA expression is significantly associated with tumor size. Patients were divided into high and low expression groups according to the median expression level of LINC01638 lncRNA. The association between LINC01638 lncRNA and clinicopathological data of patients with melanoma was analyzed using $\chi^{2}$ test. Results revealed that LINC01638 lncRNA expression in skin biopsies (Table II) and plasma (Table III) was significantly associated with tumor size but not age, sex or patients' smoking and drinking habits.
LINC01638 IncRNA expression is further upregulated in patients with local recurrence but not in patients without recurrence during follow-up. During the five year follow-up, local recurrence (cancer grows in the same region it first started) was observed in 17 cases and no distant recurrence was observed. Compared with pre-treatment levels, LINC01638 lncRNA expression was further upregulated in patients with local recurrence $(\mathrm{P}<0.05$; Fig. $3 \mathrm{~A})$, but not in patients without recurrence (Fig. 3B). Hazard ratios of tumor stages and LINC01638 lncRNA levels for local recurrence were 1.78 (mean, range, 1.07-2.91) and 2.20 (mean, range, 1.11-4.30), respectively.

LINC01638 IncRNA overexpression promotes, while knockdown inhibits cancer cell proliferation. The significant association between LINC01638 lncRNA overexpression and tumor size suggested the involvement of LINC01638 lncRNA in the growth of melanoma. In order to test this further, LINC01638 lncRNA expression vectors and shRNAs were transfected into cancer cells and cell proliferation was analyzed using the CCK- 8 assay. Following transfection, LINC01638 lncRNA overexpression (Fig. 4A) and knockdown (Fig. 4B) were achieved in two human melanoma cell lines C32 and SK-MEL-28 and a normal skin cell line CCD-1059Sk 
Table II. Association between long intergenic non-protein coding RNA 1638 long non-coding RNA expression in skin biopsies and clinicopathological data of patients with melanoma.

\begin{tabular}{lcccccc}
\hline Variable & Group & Number of cases & High-expression & Low-expression & $\chi^{2}$ & P-value \\
\hline Age (years) & $>45$ & 21 & 13 & 8 & 2.51 & 0.11 \\
\multirow{5}{*}{ Sex } & $<45$ & 19 & 7 & 12 & & \\
\multirow{4}{*}{ Primary tumor diameter } & Male & 22 & 10 & 12 & 0.40 & 0.52 \\
& Female & 18 & 10 & 8 & & \\
\multirow{4}{*}{ Drinking } & $>4 \mathrm{~mm}$ & 18 & 13 & 5 & 7.03 & 0.03 \\
\multirow{3}{*}{ Smoking } & $2-4 \mathrm{~mm}$ & 13 & 2 & 8 & & \\
& $<2 \mathrm{~mm}$ & 9 & 7 & 10 & 0.92 & 0.34 \\
& Yes & 17 & 13 & 10 & & 0.52 \\
& No & 23 & 7 & 9 & 0.42 & 0.52 \\
\hline
\end{tabular}

Table III. Association between long intergenic non-protein coding RNA 1638 long non-coding RNA expression in plasma and clinicopathological data of patients with melanoma.

\begin{tabular}{|c|c|c|c|c|c|c|}
\hline Variable & Group & Number of cases & High-expression & Low-expression & $\chi^{2}$ & P-value \\
\hline \multirow[t]{2}{*}{ Age (years) } & $>45$ & 21 & 13 & 8 & \multirow[t]{2}{*}{2.51} & \multirow[t]{2}{*}{0.11} \\
\hline & $<45$ & 19 & 7 & 12 & & \\
\hline \multirow[t]{2}{*}{ Sex } & Male & 22 & 9 & 13 & \multirow[t]{2}{*}{1.60} & \multirow[t]{2}{*}{0.20} \\
\hline & Female & 18 & 11 & 7 & & \\
\hline \multirow[t]{3}{*}{ Primary tumor diameter $(\mathrm{mm})$} & $>4$ & 18 & 13 & 5 & \multirow[t]{3}{*}{6.48} & \multirow[t]{3}{*}{0.04} \\
\hline & $2-4$ & 13 & 4 & 9 & & \\
\hline & $<2$ & 9 & 3 & 6 & & \\
\hline \multirow[t]{2}{*}{ Drinking } & Yes & 17 & 8 & 9 & \multirow[t]{2}{*}{0.10} & \multirow[t]{2}{*}{0.75} \\
\hline & No & 23 & 12 & 11 & & \\
\hline \multirow[t]{2}{*}{ Smoking } & Yes & 16 & 6 & 10 & \multirow[t]{2}{*}{1.67} & \multirow[t]{2}{*}{0.20} \\
\hline & No & 24 & 14 & 10 & & \\
\hline
\end{tabular}

A

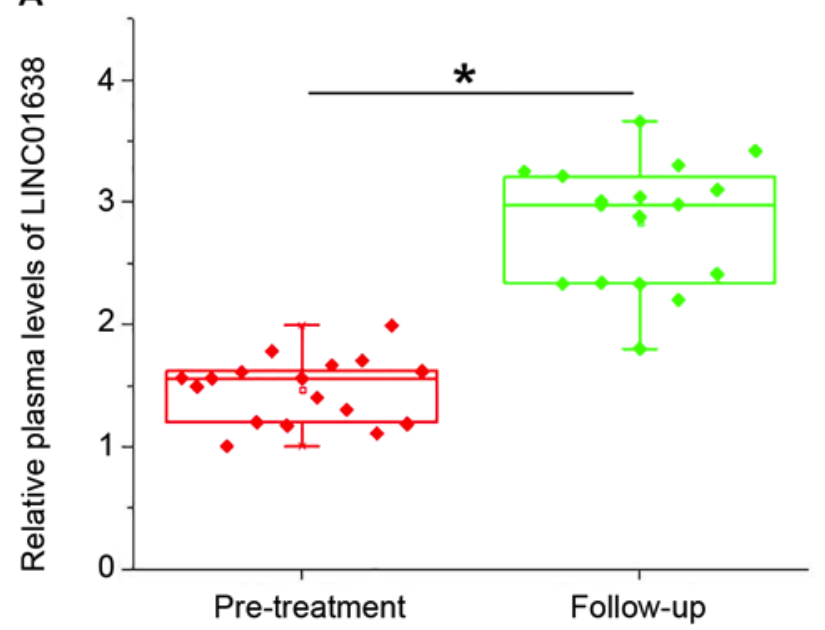

B

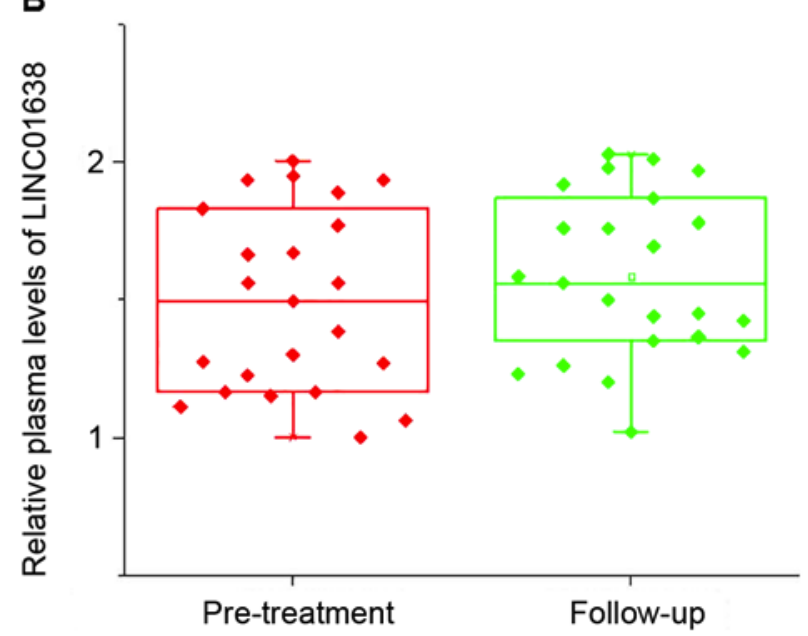

Figure 3. LINC01638 lncRNA expression is further upregulated in patients with local recurrence, however not in patients without recurrence during follow-up. LINC01638 long non-coding RNA expression (A) in patients with local recurrence and (B) patients without recurrence during the follow-up. * $\mathrm{P}<0.05$. LINC01638, long intergenic non-protein coding RNA 1638. 
A
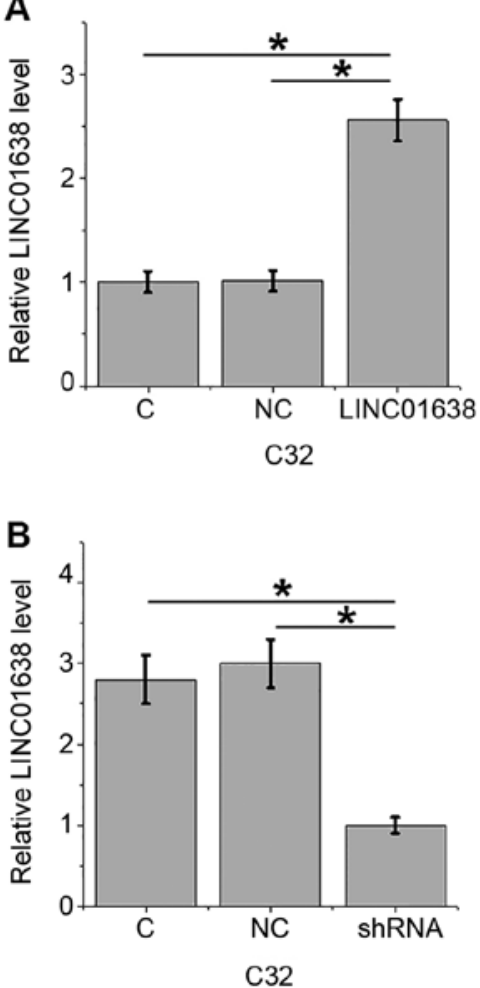
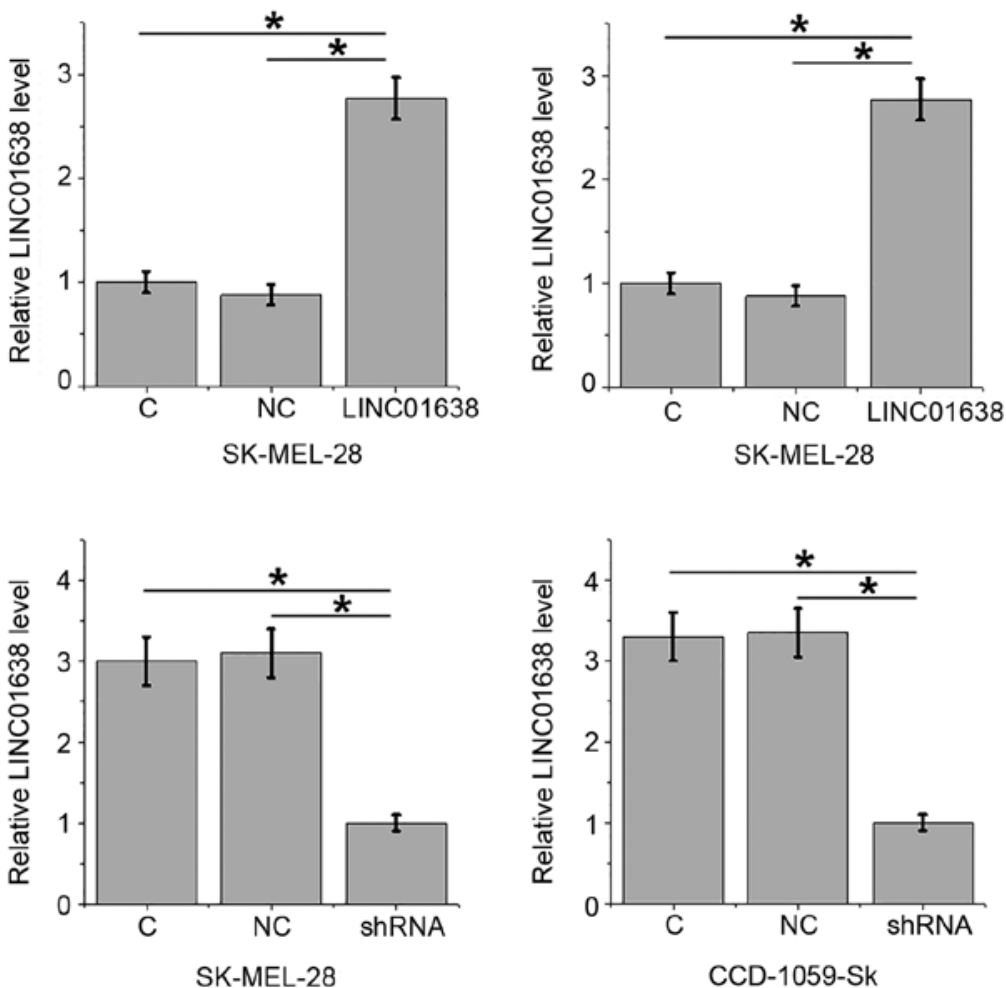

Figure 4. LINC01638 lncRNA overexpression and knockdown in cells of all three cell lines. Following transfection, LINC01638 lncRNA (A) overexpression and (B) knockdown were confirmed in cells of all three cell lines. "P<0.05. C, control, NC, negative control; shRNA, short hairpin RNA; LINC01638, long intergenic non-protein coding RNA 1638; IncRNA, long non-coding RNA.
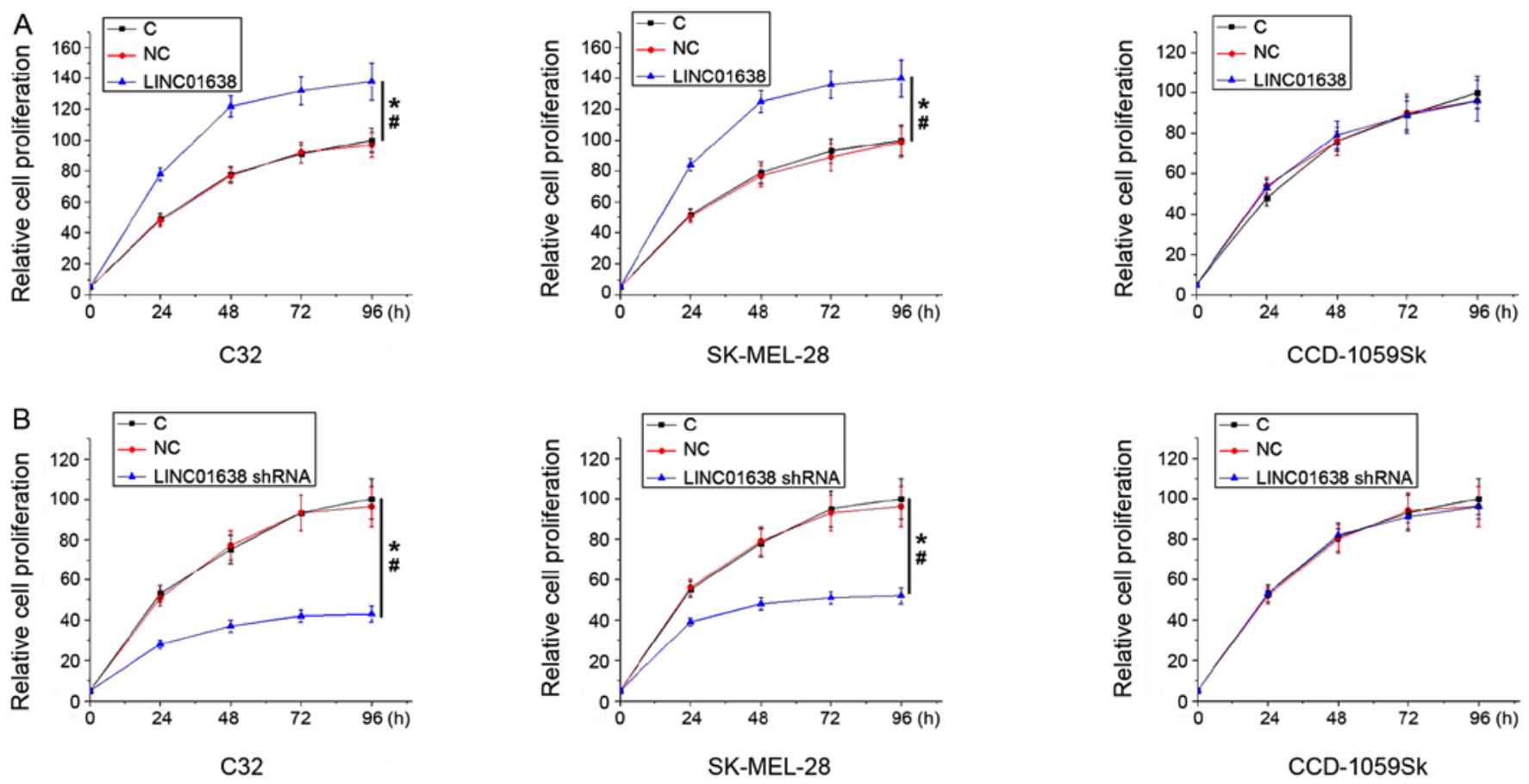

Figure 5. LINC01638 1ncRNA overexpression promotes, while knockdown inhibits cancer cell proliferation. Effect of LINC01638 lncRNA (A) overexpression (B) knockdown on cell proliferation of two human melanoma cell lines C32 and SK-MEL-28, and normal skin cell line CCD-1059Sk. ${ }^{*} \mathrm{P}<0.05$ vs C; ${ }^{*} \mathrm{P}<0.05$ vs NC;. C, control, NC, negative control; shRNA, short hairpin RNA; LINC01638, long intergenic non-protein coding RNA 1638; lncRNA, long non-coding RNA.

$(\mathrm{P}<0.05)$. Compared with control cells and negative control cells, LINC01638 lncRNA overexpression significantly promoted (Fig. 5A), while LINC01638 IncRNA knockdown significantly inhibited (Fig. 5B) cell proliferation of the two human melanoma cell lines C32 and SK-MEL-28 $(\mathrm{P}<0.05)$, but not cells of the normal skin cell line CCD-1059Sk. 


\section{Discussion}

The function of LINC01638 lncRNA has only been characterized in triple-negative breast cancer, while its involvement in other diseases is unknown (10). The results obtained in the current study suggested that LINC01638 1ncRNA is upregulated in melanoma and may participate in the growth of melanoma as well as its local recurrence following surgical resection.

In spite of efforts to treat patients with melanoma at advanced stages, the survival rates of patients with metastatic melanoma are poor $(12,13)$. At present, early diagnosis followed by proper treatment remain key for increasing the survival rate of patients with melanoma (14). LINC01638 lncRNA has been reported to be upregulated in triple-negative cancer tissues compared with adjacent healthy tissues (10). In the present study, the involvement of LINC01638 lncRNA in the early stages of melanoma was studied in patients with melanoma at AJCC stage I-IIIA. Compared with healthy controls, upregulation of LINC01638 lncRNA in biopsies and plasma was observed in patients with melanoma but not in patients with benign skin cancer. Overexpression of LINC01638 lncRNA in biopsies and plasma effectively distinguished patients with melanoma from patients with benign skin cancer and healthy controls. In addition, expression of LINC01638 lncRNA was not affected by age, sex and smoking and drinking habits, which are factors that affect the expression of certain lncRNAs $(15,16)$. This indicated that the overexpression of LINC01638 lncRNA may be consistent across patients with melanoma with different backgrounds. Therefore, detecting the expression of LINC01638 IncRNA may aid in the early diagnosis of melanoma.

In the current study, the expression of LINC01638 IncRNA was quantified in skin biopsies of patients with melanoma, patients with benign skin lesion and healthy controls. Skin biopsy is an invasive technique with low patient acceptability, and therefore, a small number of samples was included in the current study. The development of human diseases may be accompanied by changes of blood substances, and the detection of these changes may provide guidance for the treatment of melanoma $(17,18)$. Circulating lncRNAs, including Pvt1 oncogene (19) and maternally expressed 3 (20) have demonstrated diagnostic value for melanoma. In the current study, the diagnostic value of plasma LINC01638 lncNRA was comparable to that of LINC01638 $\operatorname{lncNRA}$ expression in skin biopsies. Therefore, the detection of plasma LINC01638 IncNRA may allow the diagnosis of melanoma in cases where skin biopsy is not applicable.

Accurate prediction of tumor recurrence is required for the prevention and treatment of melanoma $(21,22)$. In the present study, patients with local recurrence exhibited significantly increased plasma level of LINC01638 lncNRA during follow-up compared with patients with no recurrence, indicating that LINC01638 lncNRA overexpression may participate in the local recurrence of melanoma. Although the results obtained in the current study suggest that LINC01638 IncNRA enhances the proliferation of melanoma cells in vitro, which may promote local recurrence, the molecular mechanism underlying recurrence remains unknown. Future studies are required to elucidate the molecular mechanisms by which LINC01638 lncNRA results in the recurrence of melanoma. In the current study, LINC01638 lncRNA overexpression promoted melanoma cell proliferation, but failed to significantly affect cell migration and invasion. Therefore, LINC01638 IncRNA may specifically participate in the growth of melanoma.

In conclusion, LINC01638 lncRNA is overexpressed in patients with melanoma. Upregulation of LINC01638 lncRNA is likely to be associated with the local recurrence of melanoma following surgical resection.

\section{Acknowledgements}

Not applicable.

\section{Funding}

This study was supported by Hunan Provincial People's Hospital 2007 Renshu Fund (Changsha, China).

\section{Availability of data and materials}

The datasets used and/or analyzed during the current study are available from the corresponding author on reasonable request.

\section{Authors' contributions}

WX and AY performed all the experiments, analyzed all data. AY drafted the manuscript. Both authors read and approved the final manuscript.

\section{Ethics approval and consent to participate}

This study received ethical approval from the Hunan People's Hospital (Changsha, China) Ethics Committee. All participants signed informed consent.

\section{Patient consent for publication}

The study followed the tenets of the Declaration of Helsinki, and informed written consent was obtained from all patients and controls.

\section{Competing interests}

The authors declare that they have no competing interests.

\section{References}

1. Chen W: Cancer statistics: Updated cancer burden in China. Chin J Res 27: 1, 2015.

2. Karimkhani C, Green AC, Nijsten T, Weinstock MA, Dellavalle RP, Naghavi M and Fitzmaurice C: The global burden of melanoma: Results from the global burden of disease study 2015. Br J Dermatol 177: 134-140, 2017.

3. Tsao H, Chin L, Garraway LA and Fisher DE: Melanoma: From mutations to medicine. Genes Dev 26: 1131-1155, 2012.

4. Farma JM,Kulkarni N and Hsu C: Surgical management of primary and recurrent melanoma. Surg Oncol Clin N Am 24: 239-247, 2015.

5. Hocker T and Tsao H: Ultraviolet radiation and melanoma: A systematic review and analysis of reported sequence variants. Hum Mutat 28: 578-588, 2007. 
6. Silverberg JI and Ratner D: Associations of non-melanoma skin cancer and melanoma, extra-cutaneous cancers and smoking in adults: A US population-based study. J Eur Acad Dermatol Venereol 29: 1389-1397, 2015.

7. Esteller M: Non-coding RNAs in human disease. Nat Rev Genet 12: 861-874, 2011.

8. Mercer TR, Dinger ME and Mattick JS: Long non-coding RNAs: Insights into functions. Nat Rev Genet 10: 155-159, 2009.

9. Spizzo R, Almeida MI, Colombatti A and Calin GA: Long non-coding RNAs and cancer: A new frontier of translational research? Oncogene 31: 4577-4587, 2012.

10. Luo L, Tang H, Ling L, Li N, Jia X, Zhang Z, Wang X, Shi L, Yin J, Qiu N, et al: LINC01638 lncRNA activates MTDH-Twist1 signaling by preventing SPOP-mediated c-Myc degradation in triple-negative breast cancer. Oncogene 37: 6166-6179, 2018.

11. Livak KJ and Schmittgen TD: Analysis of relative gene expression data using real-time quantitative PCR and the 2(-Delta Delta C(T)) method. Methods 25: 402-408, 2001.

12. Hodi FS, O'Day SJ, McDermott DF, Weber RW, Sosman JA, Haanen JB, Gonzalez R, Robert C, Schadendorf D, Hassel JC, et al: Improved survival with ipilimumab in patients with metastatic melanoma. N Engl J Med 363: 711-723, 2010.

13. Schadendorf D, Hodi FS, Robert C, Weber JS, Margolin K, Hamid O, Patt D, Chen TT, Berman DM and Wolchok JD: Pooled analysis of long-term survival data from phase II and phase III trials of ipilimumab in unresectable or metastatic melanoma. J Clin Oncol 33: 1889-1894, 2015.

14. American Academy of Dermatology Ad Hoc Task Force for the ABCDEs of Melanoma, Tsao H, Olazagasti JM, Cordoro KM, Brewer JD, Taylor SC, Bordeaux JS, Chren MM, Sober AJ, Tegeler C, et al: Early detection of melanoma: Reviewing the ABCDEs. J Am Acad Dermatol 72: 717-723, 2015.

15. Grammatikakis I, Panda AC, Abdelmohsen K and Gorospe $M$ : Long noncoding RNAs(lncRNAs) and the molecular hallmarks of aging. Aging (Albany NY) 6: 992-1009, 2014.
16. Wang J, Qiu M, Xu Y, Li M, Dong G, Mao Q, Yin R and Xu L: Long noncoding RNA CCAT2 correlates with smoking in esophageal squamous cell carcinoma. Tumour Biol 36: 5523-5528, 2015.

17. Zeng L, Liu J, Wang Y, Wang L, Weng S, Tang Y, Zheng C, Cheng Q, Chen S and Yang GY: MicroRNA-210 as a novel blood biomarker in acute cerebral ischemia. Front Biosci (Elite Ed) 3: 1265-1272, 2011

18. Shehadeh LA, Yu K, Wang L, Guevara A, Singer C, Vance J and Papapetropoulos S: SRRM2, a potential blood biomarker revealing high alternative splicing in Parkinson's disease. PLoS One 5: e9104, 2010.

19. Chen X, Gao G, Liu S, Yu L, Yan D, Yao X, Sun W, Han D and Dong H: Long noncoding RNA PVT1 as a novel diagnostic biomarker and therapeutic target for melanoma. Biomed Res Int 2017: 7038579, 2017.

20. Long J and Pi X: lncRNA-MEG3 suppresses the proliferation and invasion of melanoma by regulating CYLD expression mediated by sponging miR-499-5p. Biomed Res Int 2018: $2086564,2018$.

21. Mallidi S, Watanabe K, Timerman D, Schoenfeld D and Hasan T: Prediction of tumor recurrence and therapy monitoring using ultrasound-guided photoacoustic imaging. Theranostics 5: 289-301, 2015.

22. Mattonen SA, Palma DA, Haasbeek CJ, Senan S and Ward AD: Early prediction of tumor recurrence based on CT texture changes after stereotactic ablative radiotherapy (SABR) for lung cancer. Med Phys 41: 033502, 2014.

(i) $(-)$ This work is licensed under a Creative Commons Attribution-NonCommercial-NoDerivatives 4.0 International (CC BY-NC-ND 4.0) License. 